Also available at http://amc-journal.eu

ISSN 1855-3966 (printed edn.), ISSN 1855-3974 (electronic edn.)

ARS MATHEMATICA CONTEMPORANEA 11 (2016) 91-99

\title{
Accola theorem on hyperelliptic graphs
}

\author{
Maxim P. Limonov* \\ Sobolev Institute of Mathematics, 630090, Koptyuga 4, Novosibirsk, Russia \\ Novosibirsk State University, 630090, Pirogova st. 2, Novosibirsk, Russia \\ Laboratory of Quantum Topology, Chelyabinsk State University, \\ Br. Kashirinykh str., 129, room 419, 430, 454001, Chelyabinsk, Russia
}

Received 3 January 2015, accepted 27 May 2015, published online 24 September 2015

\begin{abstract}
In this paper, we prove the following theorem: If a graph $X$ is a degree 2 (unramified) covering of a hyperelliptic graph of genus $g \geq 2$, then $X$ is $\gamma$-hyperelliptic for some $\gamma \leq\left[\frac{g-1}{2}\right]$. This is a discrete analogue of the corresponding theorem for Riemann surfaces. The Bass-Serre theory of coverings of graphs of groups is employed to get the main result.

Keywords: Riemann surface, graph, hyperelliptic graph, fundamental group, automorphism group, harmonic map, branched covering, graph of groups.
\end{abstract}

Math. Subj. Class.: 05C10, 20E08, 57M12

\section{Introduction}

Let $M$ be a compact Riemann surface and let $G$ be its finite group of conformal automorphisms, admitting a partition. That is, $G$ can be expressed as a set-theoretic union of its certain subgroups with trivial pairwise intersections. In [2], R. D. M. Accola proved a formula which relates the genera of $M, M / G$ and $M / G_{i}$ where subgroups $G_{i}, i=1,2, \ldots, s$, form a partition. This formula is as follows:

$$
(s-1) g(M)+|G| g(M / G)=\sum_{i=1}^{s}\left|G_{i}\right| g\left(M / G_{i}\right) .
$$

Demonstrating the applications of the formula, in the same paper Accola proved the following theorem, first proved by H. M. Farkas [7] using theta functions: If $M$ is a compact

* The work was partially supported by Laboratory of Quantum Topology of Chelyabinsk State University (Russian Federation government grant 14.Z50.31.0020), Russian Foundation for Basic Research (grant 15-0107906), Leading Scientific Schools Support Program (grant SS-2263.2014.1) and by the Slovenian-Russian grant (2014-2015).

E-mail address: volsterm@gmail.com (Maxim P. Limonov)

(a) (i) This work is licensed under http://creativecommons.org/licenses/by/3.0/ 
Riemann surface of genus three which is a two-fold unramified covering of a genus $g=2$ hyperelliptic Riemann surface, then $M$ is hyperelliptic. The case $g>2$ was considered in papers [1], [5]. For example, in the case of $g=3$ it turns out that $M$ is hyperelliptic or 1-hyperelliptic ( $M$ is a two-fold covering of a torus).

In this paper, we find a discrete version of results obtained in [1] and [5]. Finite connected graphs here play the role of Riemann surfaces, and harmonic maps between graphs play the role of holomorphic maps between Riemann surfaces. It turns out that the category of graphs, together with harmonic maps between them, closely mirrors the category of Riemann surfaces, together with the holomorphic maps between them. Namely, we prove that if a graph $X$ is a degree 2 (unramified) covering of a hyperelliptic graph $Y$ of genus $g \geq 2$, then $X$ is $\gamma$-hyperelliptic for some $\gamma \leq\left[\frac{g-1}{2}\right]$.

Graph $X$, from the statement above, has the property that its automorphism group contains the Klein four-subgroup. In the proof, we use the fact that the Klein four-group admits a partition, and apply an analogue of (1.1) from [14].

Also we employ the theory of graphs of groups (or the Bass-Serre theory) to uniformize the coverings of a graph just as it works for Riemann surfaces. This approach was proposed by A. Mednykh and I. Mednykh [12].

In his dissertation [8], M. T. Green generalized the Bass-Serre theory and for coverings of graphs of groups obtained results similar to those in the topological theory of coverings. We use some results from this $\mathrm{Ph} . \mathrm{D}$. thesis.

\section{Preliminaries}

\subsection{Graphs}

In the present paper, a graph is a finite connected multigraph. We allow a graph to have loops. Denote by $V(X)$ the set of vertices of $X$ and by $E(X)$ the set of directed edges of $X$. Following J.-P. Serre [13], we introduce two maps $\partial_{0}, \partial_{1}: E(X) \rightarrow V(X)$ (endpoints) and a fixed point free involution $e \rightarrow \bar{e}$ of $E(X)$ (reversal of orientation) such that $\partial_{i} \bar{e}=$ $\partial_{1-i} e$. We put

$$
\mathrm{St}(a)=\mathrm{St}^{X}(a)=\partial_{0}^{-1}(a)=\left\{e \in E(X) \mid \partial_{0} e=a\right\},
$$

the star of $a$, and call $\operatorname{deg}(a)=|S t(a)|$ the degree (or valency) of $a$. A morphism of graphs $\varphi: X \rightarrow Y$ carries vertices to vertices, edges to edges, and, for $e \in E(X), \varphi\left(\partial_{i} e\right)=$ $\partial_{i} \varphi(e)(i=0,1)$ and $\varphi(\bar{e})=\overline{\varphi(e)}$. Note that a morphism of graphs carries loops to loops. Working with loops in a graph, one may encounter some problems. On those occasions, one can use the approach with semiedges being developed in [9].

For $a \in X$ we have the local map

$$
\varphi_{a}: \mathrm{St}^{X}(a) \rightarrow \mathrm{St}^{Y}(\varphi(a)) .
$$

A map $\varphi$ is locally bijective if $\varphi_{a}$ is bijective for all $a \in X$. We call $\varphi$ a covering if $\varphi$ is surjective and locally bijective. A bijective morphism is called an isomorphism, and an isomorphism $\varphi: X \rightarrow X$ is called an automorphism.

Remark 2.1. Note that the definition of a morphism of graphs given by M. Baker and $\mathrm{S}$. Norine in [3] and our definition differ in the following sense. Let $\varphi: X \rightarrow Y$ be morphism of graphs and for some edge $e \in E(X)$ let $\varphi\left(\partial_{0} e\right)=\varphi\left(\partial_{1} e\right)=b \in V(Y)$. Then morphism $\varphi$, in the sense of [3], sends edge $e$ to vertex $b$. In our case, morphism $\varphi$ must send edge $e$ to a loop based at vertex $b$. 


\subsection{Harmonic maps and harmonic actions}

In this paragraph, we specify the class of morphisms of graphs, called harmonic maps, that share most properties with holomorphic maps between Riemann surfaces. The notion of harmonic maps between graphs was introduced by H. Urakawa [15] for simple graphs and was generalized by M. Baker and S. Norine [3] for multigraphs.

Definition 2.2. A morphism $\varphi: X \rightarrow Y$ of graphs is said to be a harmonic map or branched covering if, for all $x \in V(X), y \in V(Y)$ such that $y=\varphi(x)$, the quantity

$$
\left|e \in E(X): x=\partial_{0} e, \varphi(e)=e^{\prime}\right|
$$

is the same for all edges $e^{\prime} \in E(Y)$ such that $y=\partial_{0} e^{\prime}$.

One can check directly from the definition that the composition of two harmonic morphisms is again harmonic. Therefore the class of all graphs, together with the harmonic morphisms between them, forms a category. We note also that an arbitrary covering of graphs is a harmonic map.

Let $\varphi: X \rightarrow Y$ be harmonic and $x \in V(X)$. We define the multiplicity of $\varphi$ at $x$ by

$$
m_{\varphi}(x)=\left|e \in E(X): x=\partial_{0} e, \varphi(e)=e^{\prime}\right|
$$

for any edge $e^{\prime} \in E(X)$ such that $\varphi(x)=\partial_{0} e^{\prime}$. By the definition of a harmonic morphism, $m_{\varphi}(x)$ is independent of the choice of $e^{\prime}$. If $m_{\varphi}(x)>1$ for some vertex $x \in V(X)$, such a vertex is called a ramification point of $\varphi$. The image $\varphi(x)$ of a ramification point is called a branch point.

We define the degree of a harmonic map $\varphi: X \rightarrow Y$ by the formula

$$
\operatorname{deg}(\varphi):=\left|e \in E(X): \varphi(e)=e^{\prime}\right|
$$

for any edge $e^{\prime} \in E(Y)$. From the definition of a harmonic map of graphs and connectivity of the graphs, it follows that the right-hand side of (2.1) does not depend on the choice of $e^{\prime}$ and therefore $\operatorname{deg}(\varphi)$ is well defined.

Let $G<\operatorname{Aut}(X)$ be a group of automorphisms of a graph $X$. An edge $e \in E(X)$ is called invertible if there is $h \in G$ such that $h(e)=\bar{e}$. Let $G$ act without invertible edges. Define the quotient graph $X / G$ so that its vertices and edges are $G$-orbits of the vertices and edges of $X$. Note that if the endpoints of an edge $e \in E(X)$ lie in the same $G$-orbit then the $G$-orbit of $e$ is a loop in the quotient graph $X / G$. Following S. Corry [6], we say that the group $G$ acts harmonically on a graph $X$ if for all subgroups $H<G$, the canonical projection $\varphi_{H}: X \rightarrow X / H$ is harmonic. If $G$ acts harmonically and without invertible edges, we say that $G$ acts purely harmonically on $X$.

The genus of a graph is defined as the rank of the first homology group of the graph (that is, its cyclomatic number). Let $X$ be a graph of genus $g^{\prime}$ and let a group $G<\operatorname{Aut}(X)$ act purely harmonically on $X$. Denote by $g$ the genus of the quotient graph $X / G$. There is an analogue of the Riemann-Hurwitz relation for graphs introduced in [3]. For the graph morphism under consideration, the relation is proved in [11], and has the following form:

$$
g^{\prime}-1=|G|(g-1)+\sum_{a \in V(X)}\left(\left|G^{a}\right|-1\right),
$$

where $G^{a}$ stands for the stabilizer of $a \in V(X)$. Here $|G|$ coincides with the degree of the harmonic map $\varphi: X \rightarrow X / G$ and $\left|G^{a}\right|$ coincides with the multiplicity $m_{\varphi}(a)$ of $\varphi$ at $a$. 
Remark 2.3. A graph $X$ of genus $g^{\prime} \geq 2$ is said to be hyperelliptic, if there is a degree 2 harmonic map $F: X \rightarrow Y$, where graph $Y$ is a tree (that is, a graph of genus 0 ). Since at every ramification point $x \in V(X)$ the multiplicity $m_{F}(x)=2$, by (2.2) the number of ramification points of $F$ is equal to $g^{\prime}+1$.

A finite group $G$ is said to admit a partition $\left\{G_{1}, \ldots, G_{s}\right\}$, where $G_{i}<G$ and $s \geq 2$, if $G=\bigcup_{i=1}^{s} G_{i}$ and $G_{i} \cap G_{j}=\{1\}, i, j=1,2, \ldots, s, i \neq j$. Let $G<\operatorname{Aut}(X)$ act purely harmonically on a graph $X$ and admit a partition $\left\{G_{1}, \cdots, G_{s}\right\}$. Recall that the Euler characteristic $\chi(X)$ of a graph $X$ is related to the genus $g(X)$ of $X$ via $\chi(X)=1-g(X)$. By Corollary 1 in [14], we have

$$
(s-1) g(X)+|G| g(X / G)=\sum_{i=1}^{s}\left|G_{i}\right| g\left(X / G_{i}\right) .
$$

\subsection{Graphs of groups}

The theory of graphs of groups is employed in this paper to uniformize harmonic maps between graphs. Following [4], we give the definition.

Definition 2.4. A graph of groups $\mathbb{X}=(X, \mathcal{A})$ consists of

(i) a connected graph $X$;

(ii) an assignment $\mathcal{A}$ to every vertex $a \in V(X)$ a group $\mathcal{A}_{a}$, and to every edge $e \in E(X)$ a group $\mathcal{A}_{e}=\mathcal{A}_{\bar{e}}$;

(iii) monomorphisms $\alpha_{e}: \mathcal{A}_{e} \rightarrow \mathcal{A}_{a}$, where $a=\partial_{0} e$.

In this paper we restrict ourselves to a class of graphs of groups having trivial groups $\mathcal{A}_{e}=\{1\}$ for all edges $e \in E(X)$ and finite groups $\mathcal{A}_{a}$ for all vertices $a \in V(X)$. It will be enough for application to the theory of harmonic maps between graphs.

There are two equivalent definitions of the notion of a fundamental group of a graph of groups: the first is a direct algebraic definition via an explicit group presentation, and the second one using the language of groupoids. The algebraic definition is easier to state. Choose a spanning tree $T$ in $X$. The fundamental group of $X$ with respect to $T$, denoted $\pi_{1}(\mathbb{X}, T)$, is defined as the quotient of the free product

$$
\left[\left(\underset{a \in V(X)}{*} \mathcal{A}_{a}\right) * F(E(X))\right] / R
$$

where $F(E(X))$ denotes the free group with basis $E(X)$ and $R$ is the following set of relations:

(i) $\bar{e}=e^{-1}$ for every $e$ in $E(X)$;

(ii) $e=1$ for every $e$ in $E(T)$.

There is also a notion of the fundamental group of $\mathbb{X}$ with respect to a base-vertex $a$ in $X$, denoted $\pi_{1}(\mathbb{X}, a)$, which is defined using the formalism of groupoids (see [8] and [4] for details). It turns out that for every choice of a base-vertex $a$ and every spanning tree $T$ in $X$, the groups $\pi_{1}(\mathbb{X}, T)$ and $\pi_{1}(\mathbb{X}, a)$ are naturally isomorphic. We note also ([4], 
section 1.22) that for given $a, b \in X$ the groups $\pi_{1}(\mathbb{X}, a)$ and $\pi_{1}(\mathbb{X}, b)$ are conjugate in the fundamental groupoid of $\mathbb{X}$. In what follows we will use notation $\pi_{1}(\mathbb{X})$, ignoring the way the fundamental group was constructed.

It follows from the above definition that if $X$ is a graph of genus $g$ then $F(E(X)) / R=$ $F_{g}$ is the free group of rank $g$. Then

$$
\pi_{1}(\mathbb{X})=\left(\underset{a \in V(X)}{*} \mathcal{A}_{a}\right) * F_{g}
$$

To every graph of groups $\mathbb{X}$, with a specified choice of a base-vertex $a \in X$, one can associate a Bass-Serre universal covering tree $\widetilde{\mathbb{X}}=\widetilde{(\mathbb{X}, a)}$, which is a tree admitting a natural group action of the fundamental group $\pi_{1}(\mathbb{X})=\pi_{1}(\mathbb{X}, a)$ without edge-inversions. Moreover, the quotient graph of groups $\widetilde{\mathbb{X}} / \pi_{1}(\mathbb{X})$ is naturally isomorphic to $\mathbb{X}$.

\subsection{Coverings of graphs of groups and harmonic maps}

Let us take graph morphisms in the definition of a covering of graphs of groups, given in [8] or [4], to be the class of all harmonic maps. Taking into consideration the fact that a trivial group is assigned to any edge, the definition of a covering of graphs of groups can be formulated as follows.

Definition 2.5. Let $\mathbb{X}=(X, \mathcal{A})$ and $\mathbb{Y}=(Y, \mathcal{B})$ be graphs of groups with trivial edge groups. A covering $\mathbb{F}=(F, \Phi): \mathbb{X} \rightarrow \mathbb{Y}$ of graphs of groups consists of

(i) a harmonic morphism $F: X \rightarrow Y$;

(ii) a set $\Phi$ of monomorphisms $F_{a}: \mathcal{A}_{a} \rightarrow \mathcal{B}_{F(a)}, a \in V(X)$, such that $m_{F}(a)\left|\mathcal{A}_{a}\right|=\left|\mathcal{B}_{F(a)}\right|$, where $m_{F}(a)$ is the multiplicity of $F$ at the point $a$.

This definition was introduced in [12]. To illustrate the notion of a covering in the category of graphs of groups, we provide a basic example.

Example 2.6. Let $G$ be a group of automorphisms of a finite connected graph $X$. Suppose that $G$ acts on the set $E(X)$ of directed edges of $X$ freely and without edge inversions. Consider the canonical map $F: X \rightarrow Y=X / G$. Denote by $\operatorname{St}_{G}(a)$ the stabilizer of a vertex $a$ in group $G$. Then $F$ is a harmonic map with $m_{F}(a)=\left|\operatorname{St}_{G}(a)\right|, a \in V(X)$. Denote by $\mathbb{X}$ the graph of groups obtained from $X$ by prescribing a trivial group to each vertex and each edge of $X$. Graph of groups $\mathbb{Y}$ is defined by prescribing to each vertex $b=F(a)$ of $Y$ a group $\mathcal{B}_{F(a)}$ isomorphic to $\operatorname{St}_{G}(a)$ and assign a trivial group to each edge of $Y$. Since $G$ acts transitively on each fibre of $F$, the group $\mathcal{B}_{F(a)}$ is well defined. Let $\Phi$ be the set of trivial monomorphisms $F_{a}: \mathcal{A}_{a} \rightarrow \mathcal{B}_{F(a)}, a \in V(X)$. We have $m_{F}(a)\left|\mathcal{A}_{a}\right|=\left|\mathcal{B}_{F(a)}\right|$. Then

$$
\mathbb{F}=(F, \Phi): \mathbb{X} \rightarrow \mathbb{Y}=\mathbb{X} / G
$$

is the covering of graphs of groups. 


\section{Main result}

A graph $X$ of genus $g^{\prime} \geq 2$ is said to be $\gamma$-hyperelliptic if there is a degree 2 harmonic map $F: X \rightarrow Y$ onto a graph $Y$ of genus $\gamma$. Each edge of $Y$ has two pre-images under $F$ and there is an order 2 automorphism $\tau$ of $X$, which swaps these pre-images. This automorphism is called $\gamma$-hyperelliptic involution. Note that $\gamma$-hyperelliptic involution acts on $X$ purely harmonically. The case $\gamma=0$ coincides with the definition of a hyperelliptic graph. The main result is stated in the following theorem.

Theorem 3.1. Let $X$ be a degree 2 (unramified) covering of a hyperelliptic graph $Y$ of genus $g \geq 2$. Then $X$ is $\gamma$-hyperelliptic for some $\gamma \leq\left[\frac{g-1}{2}\right]$.

In the proof of Theorem 3.1 we use the following algebraic result.

Lemma 3.2. Let $\Gamma$ be a free product of $n>1$ copies of $\mathbb{Z}_{2}$. If $F<\Gamma$ is a torsion-free subgroup of index 4 , then $F \triangleleft \Gamma$ and $\Gamma / F$ is isomorphic to the Klein four-group.

Proof. The given group $\Gamma$ has the presentation

$$
\Gamma=\left\langle x_{1}, x_{2}, \ldots, x_{n} \mid x_{1}^{2}, x_{2}^{2}, \ldots, x_{n}^{2}\right\rangle .
$$

Let $F \leq \Gamma$ be any torsion-free subgroup of index 4 . The action of $\Gamma$ on the right cosets $\left\{F, F y_{1}, F y_{2}, F y_{3}\right\}$ of $F$ in $\Gamma$ gives a transitive representation $\theta: \Gamma \rightarrow S_{4}$. If some $x_{i}$ in the presentation (3.1) of $\Gamma$ has a fixed point, then for some $y \in \Gamma$ we have $y x_{i} y^{-1} \in F$ and $F$ is not torsion-free, because $\left(y x_{i} y^{-1}\right)^{2}=1$. Hence $x_{i}$ has no fixed points, so it is represented in $S_{4}$ by a double transposition (that is, by a permutation of cyclic type (22)). So long as we deal with the transitive representation, we get an epimorphism $\theta: \Gamma \rightarrow V_{4}$, where $V_{4}$ is the Klein four-group.

Let us show that $F \leq \operatorname{ker} \theta$. Take any $w \in F$. Since $w$ fixes the coset $F$, and there are only double transposition actions and the trivial action, $w$ must fix the remaining cosets. So, $w \in \operatorname{ker} \theta$.

The reverse inclusion $\operatorname{ker} \theta \leq F$ is obvious. Thus, we get $F=\operatorname{ker} \theta \triangleleft \Gamma$.

Proof of Theorem 3.1. Let $\phi: X \rightarrow Y$ denote the covering from the theorem. The graph $Y$ is hyperelliptic, that is, there is an order two harmonic automorphism $\tau \in \operatorname{Aut}(Y)$, such that the factor graph $T=Y /\langle\tau\rangle$ is a tree. Let $\psi: Y \rightarrow T$ be the corresponding harmonic map. Let $F$ stand for the composite harmonic map $\psi \circ \phi$.

Now we are going to find a group $G_{0}$ of deck transformations of the harmonic map $F: X \rightarrow T$. To do that, we apply the Bass-Serre theory. Turn graphs $X$ and $T$ into graphs of groups as follows. Let $\mathbb{X}=(X, \mathcal{A})$ be a graph of groups based on graph $X$, and where $\mathcal{A}$ assigns a trivial group $\mathcal{A}_{z}=\{1\}$ to each vertex and each edge $z$ of $X$. Let $\mathbb{T}=(T, \mathcal{B})$ be a graph of groups based on tree $T$, and where $\mathcal{B}$ assigns the group $\mathcal{B}_{z}=\mathbb{Z}_{2}$ to each of $g+1$ branch points $z$ of map $\psi$, and a trivial group $\mathcal{B}_{z}=\{1\}$ to every other vertex and edge $z$ of $T$.

Let us show that the map $F: X \rightarrow T$ can be extended to the covering $\mathbb{F}: \mathbb{X} \rightarrow \mathbb{T}$ of graph of groups. Since $F$ is harmonic, it remains to check that, for any $a \in V(X)$, the trivial monomorphism $\mathcal{A}_{a} \rightarrow \mathcal{B}_{F(a)}$ satisfies the condition $m_{F}(a)\left|\mathcal{A}_{a}\right|=\left|\mathcal{B}_{F(a)}\right|$ or, since all $\mathcal{A}_{a}=\{1\}$, the condition

$$
m_{F}(a)=\left|\mathcal{B}_{F(a)}\right|
$$


The map $\phi$ is a covering, and so locally bijective. Hence, for any $a \in V(X), m_{F}(a)=$ $m_{\psi}(\phi(a))$. If $\phi(a)$ is a ramification point of $\psi$, then $m_{\psi}(\phi(a))=2, \mathcal{B}_{F(a)}=\mathbb{Z}_{2}$, and so (3.2) is correct. If $\phi(a)$ is not a ramification point of $\psi$, then $m_{\psi}(\phi(a))=1, \mathcal{B}_{F(a)}$ is trivial, hence (3.2) is correct as well.

Let $H=\pi_{1}(\mathbb{X})$ and $\Gamma=\pi_{1}(\mathbb{T})$ be fundamental groups, and $\widetilde{\mathbb{X}}$ and $\widetilde{\mathbb{T}}$ be universal covering trees of graphs of groups $\mathbb{X}$ and $\mathbb{T}$ respectively. Note that since $\phi$ has no ramification points, by the Riemann-Hurwitz relation (2.2), $X$ has genus $2 g-1$ and so $H$ is a free group on $2 g-1$ generators; group $\Gamma$ is a free product of $g+1$ copies of $\mathbb{Z}_{2}$.

By the Bass uniformization theorem ([3], Proposition 2.4) there exists a lift of $\mathbb{F}$ to an isomorphism $\widetilde{\mathbb{F}}: \widetilde{\mathbb{X}} \rightarrow \widetilde{\mathbb{T}}$ between the covering trees equivariant under the action of $H$ and $\Gamma$ on $\widetilde{\mathbb{X}}$ and $\widetilde{\mathbb{T}}$ respectively. Note that $\mathbb{X} \cong \widetilde{\mathbb{X}} / H$ and $\mathbb{T} \cong \widetilde{\mathbb{T}} / \Gamma$. Identifying $\widetilde{\mathbb{X}}$ and $\widetilde{\mathbb{T}}$ via $\widetilde{\mathbb{F}}$ we replace the covering $\mathbb{F}: \mathbb{X} \rightarrow \mathrm{T}$ by the covering $\mathbb{F}^{\prime}: \widetilde{\mathbb{X}} / \mathrm{H} \rightarrow \widetilde{\mathbb{X}} / \Gamma$ induced by the group inclusion $H<\Gamma$, where $H$ is of index 4 in $\Gamma$. By Lemma 3.2, since any free group is a torsion-free group, $H$ is a normal subgroup in $\Gamma$. Therefore, by Theorem 8.1 in [8], covering $\mathbb{F}^{\prime}$ is regular and its covering transformation group is $G_{0}=\Gamma / H$. Returning to the category of graphs, we get the underlying harmonic map of graphs $X \rightarrow X / G_{0}$ coinciding with $F: X \rightarrow T$ where $X / G_{0} \cong T$. Group $G_{0}$ is isomorphic to the Klein four-group. So it admits a partition $\left\{G_{1}, G_{2}, G_{3}\right\}$ into three subgroups of order two. Note that every subgroup $G_{i} \leq G_{0}$ corresponds to a harmonic map $X \rightarrow X / G_{i}$ and one of $X / G_{i}$ is isomorphic to $Y$. Let $g^{\prime}$ and $g_{i}$ be the genera of $X$ and $X / G_{i}, i \in\{0,1,2,3\}$, respectively. By (2.3) we have

$$
g^{\prime}+2 g_{0}=g_{1}+g_{2}+g_{3}
$$

Here $g_{0}=0, g^{\prime}=2 g-1$ and one of $g_{i}$ must be $g$, so we get

$$
g-1=g_{1}+g_{2}
$$

The possible cases for $g_{1}$ and $g_{2}$ (up to a symmetry) are

\begin{tabular}{c|c}
$g_{1}$ & $g_{2}$ \\
\hline 0 & $g-1$ \\
1 & $g-2$ \\
2 & $g-3$ \\
$\cdots$ & $\cdots$ \\
{$\left[\frac{g-1}{2}\right]$} & {$\left[\frac{g-1}{2}\right](+1$, if $g$ is even $)$.}
\end{tabular}

Taking $\gamma$ to be the minimum of $g_{1}$ and $g_{2}$ in each case, we get that $X$ is $\gamma$-hyperelliptic for some $\gamma \leq\left[\frac{g-1}{2}\right]$.

Finally, we show that the bound is sharp. That is, for any $g \geq 2$ there exists a graph $X$ of genus $2 g-1$, and the smallest genus of graphs $Y$, such that $X \rightarrow Y$ is a degree 2 harmonic morphism, is equal to $\left[\frac{g-1}{2}\right]$. Let $g$ be odd. Consider graph $X_{1}$ of genus $2 g-1$, depicted on Figure 1 in the case $g=5$. Its automorphism group contains five involutions. Their actions on $X_{1}$ are horizontal and vertical flips, $h, v$, two diagonal flips, $d_{1}, d_{2}$, and the rotation $r$ on $\pi$ around the center of the graph. The corresponding factor-graphs have genera $\frac{g-1}{2}, \frac{g-1}{2}, g-1, g-1$ and $g$ respectively. 
Now let $g$ be even. Consider graph $X_{2}$ of genus $2 g-1$, depicted on Figure 2 in the case $g=6$. Its automorphism group contains three involutions. They act on $X_{2}$ as horizontal and vertical flips, $h, v$, and the rotation $r$ on $\pi$ around the center of the graph.
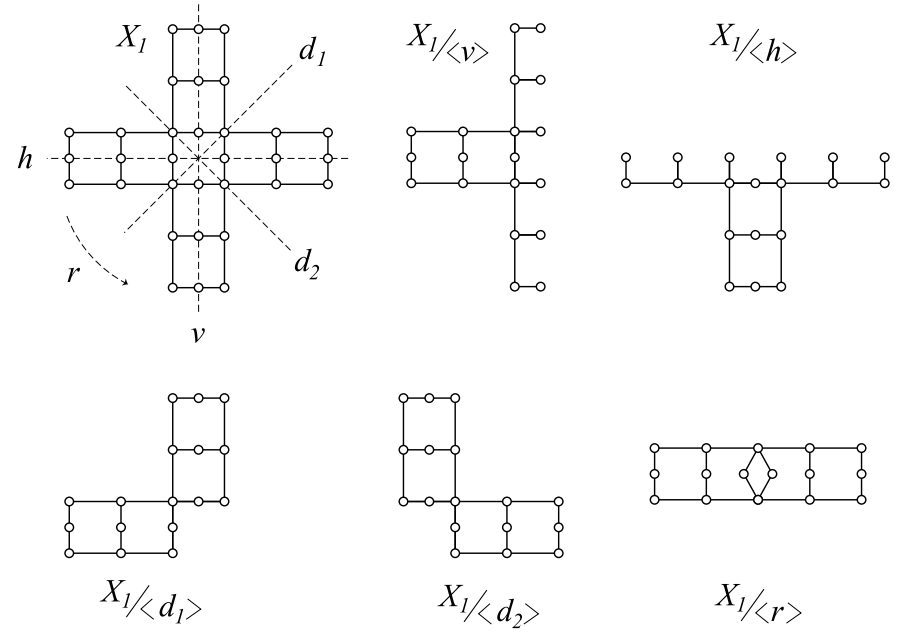

$X_{1} /\langle r\rangle$

Figure 1: Graph $X_{1}$ in the case $g=5$ and its factor-graphs.

The corresponding factor-graphs have genera $\left[\frac{g-1}{2}\right],\left[\frac{g-1}{2}\right]+1$ and $g$ respectively. Hence, the bound in the theorem is sharp.
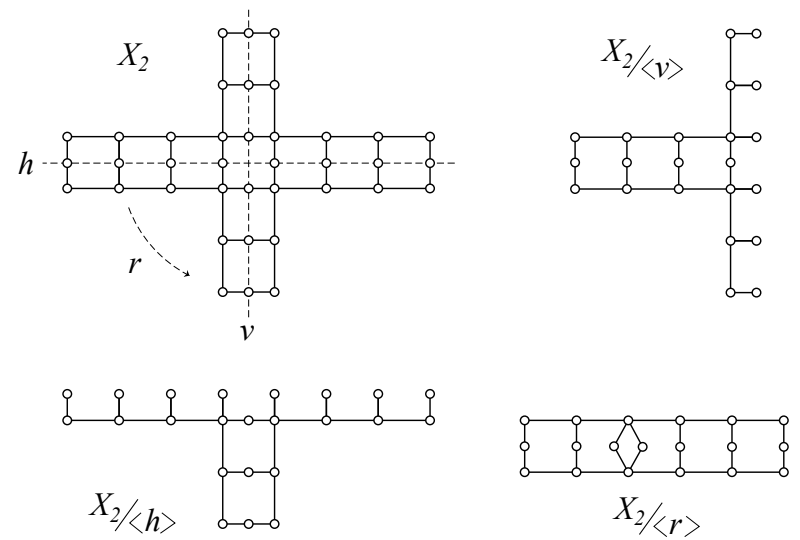

Figure 2: Graph $X_{2}$ in the case $g=6$ and its factor-graphs.

The immediate consequences of the theorem are the assertions below. The first one has been proved by I. Mednykh [10] by exhaustive search.

Corollary 3.3. Suppose $X$ is a graph of genus 3 which is a degree 2 (unramified) covering of a hyperelliptic graph $Y$ of genus 2 . Then $X$ is hyperelliptic. 
Corollary 3.4. If $X$ is a graph of genus 5 which is a degree 2 (unramified) covering of a hyperelliptic graph of genus 3 , then $X$ is hyperelliptic or 1-hyperelliptic.

Remark 3.5. In both corollaries, the genus of $X$ is not an extra hypothesis, but a necessary consequence of the degree 2 cover due to Riemann-Hurwitz relation (2.2).

\section{Acknowledgements}

The author is very grateful to his supervisor, Alexander Mednykh, and Gareth Jones for discussion of the ideas of the paper. Also the author is very grateful to an anonymous referee for fruitful remarks and suggestions.

\section{References}

[1] R. D. M. Accola, On lifting the hyperelliptic involution, Proc. Amer. Math. Soc. 122:2 (1994), 341-347.

[2] R. D. M. Accola, Riemann Surfaces with Automorphism Groups Admitting Partitions, Proc. Amer. Math. Soc. 21:2 (1969), 477-482.

[3] M. Baker, S. Norine, Harmonic morphisms and hyperelliptic graphs, Int. Math. Res. Notes 15 (2009), 2914-2955.

[4] H. Bass, Covering theory for graphs of groups, Journal of Pure and Applied Algebra 89 (1993), 3-47.

[5] E. Bujalance, A classification of unramified double coverings of hyperelliptic Riemann surfaces, Arch. Math., 47 (1986), 93-96.

[6] S. Corry, Genus bounds for harmonic group actions on finite graphs, Int. Math. Res. Notices 19 (2011), 4515-4533.

[7] H. M. Farkas, Automorphisms of compact Riemann surfaces and the vanishing of theta constants, Bull. Amer. Math. Soc. 73 (1967), 231-232.

[8] M. T. Green, Graphs of groups, Ph. D. Thesis, Department of Mathematics in the Graduate School of The University of Alabama, Tuscaloosa, 2012.

[9] A. Malnič, R. Nedela, M. Škoviera, Lifting graph automorphisms by voltage assignments, European Journal of Combinatorics, $21: 7$ (2000), 927-947.

[10] I. A. Mednykh, On the Farkas and Accola Theorems for Graphs, Doklady Mathematics, 87:1 (2013), 65-68.

[11] A.D. Mednykh, On the Riemann-Hurwitz formula for graph coverings, (2015), preprint arXiv:1505.00321v1 [math.AT]

[12] A. Mednykh, I. Mednykh, On Wiman's theorem for graphs, Discrete Mathematics (to be published).

[13] J.-P. Serre, Trees, Springer-Verlag, New York, 1980.

[14] T. Taniguchi, On the Euler-characteristic and the signature of G-manifolds, Proc. Japan Acad. 49:2 (1973), 130-133.

[15] H. Urakawa, A discrete analogue of the harmonic morphism and Green kernel comparison theorems, Glasg. Math. J., 42:3 (2000), 319-334. 